\title{
Apprehension of the disease by patients suffering from psoriasis
}

\author{
Adam Reich ${ }^{1}$, Kalina Welz-Kubiak ${ }^{1}$ tukasz Rams²
}

\author{
${ }^{1}$ Department of Dermatology, Venereology and Allergology, Wroclaw Medical University, Wroclaw, Poland \\ Head of Department: Prof. Jacek Szepietowski MD, PhD \\ ${ }^{2}$ Students' Scientific Circle of Experimental Dermatology, Department of Dermatology, Venereology and Allergology, \\ Wroclaw Medical University, Wroclaw, Poland \\ Head of Department: Prof. Jacek Szepietowski MD, PhD
}

Postep Derm Alergol 2014; XXXI, 5: 289-293

DOI: $10.5114 /$ pdia.2014.44010

\begin{abstract}
Introduction: Psoriasis affects about $1-2 \%$ of the entire population. Due to its chronicity and relapsing course, psoriasis has a great influence on patients' quality of life and psychological status.

Aim: To evaluate apprehensaion of the disease by psoriatic patients.

Material and methods: One hundred psoriasis patients (36 females, 64 males; mean age $47.3 \pm 15.8$ years) were enrolled. Mean psoriasis severity assessed according to PASI was $17.1 \pm 10.0$ points. Each participant underwent a careful physical examination and completed a specially designed questionnaire containing questions about perception of psoriasis severity, disease aggravating factors, most bothersome symptoms, possible causes of psoriasis and treatment efficacy.

Results: A correlation between patients' psoriasis assessment and objective measurement of disease intensity by PASI was weak, albeit significant $(\rho=0.37, p<0.001)$. A total of $49 \%$ of patients indicated that psoriasis had an enormous negative impact on their life and $40 \%$ declared that psoriasis decreased their self-esteem. Patients with decreased self-esteem were significantly younger and more frequently employed. A marked portion of patients believed that their disease will be cured in the future. Patients expecting a rapid cure of disease had experienced a later disease onset and suffered from psoriasis significantly shorter. The most burdensome symptoms of psoriasis were intense epidermal scaling (66\% of responders), itching (65\%), skin redness (51\%), burning (44\%), dandruff (38\%), and nail abnormalities (37\%).

Conclusions: Psoriasis may negatively affect patients' everyday life, but the degree of that influence and the level of psoriasis understanding depend on various clinical parameters as well as on demographic characteristics.
\end{abstract}

Key words: patients' beliefs, psoriasis, quality of life.

\section{Introduction}

Psoriasis is a chronic, relapsing skin disease involving $1-2 \%$ of the human population worldwide. It has a great impact on patients' everyday life, especially regarding their quality and psychosocial well-being [1]. Although a number of treatment options exist, the disease remains incurable and many previously performed studies revealed a widespread dissatisfaction as well as frustration with past and current management strategies among subjects suffering from psoriasis [1-3]. Many psoriatic individuals also feel stigmatized by the disease due to visibility of the skin lesions [4]. However, stigmatization is not only caused by the presence of psoriatic lesions on exposed body areas, but also by such symptoms as itch, pain, burning, and joint stiffness.

A low quality of life level, stigmatization and dissatisfaction with antipsoriatic therapy often lead to poor compliance with the treatment recommendations, which contributes to poor treatment efficacy, inability to fulfill the patient's expectations and further decrease in the patient's well-being. Poor compliance and adherence to therapy is also frequently related to false patient's beliefs about the disease origin, course, duration as well as treatment options and action [5]. Thus, we performed a study evaluating apprehension of the disease by pso-

Address for correspondence: Adam Reich MD, PhD, Department of Dermatology, Venereology and Allergology, Wroclaw Medical University, 1 Chalubinskiego St, 50-368 Wroclaw, Poland, phone: +48 7178422 92, fax: +48 7132709 99, e-mail: adam.reich@umed.wroc.pl Received: 7.01.2014, accepted: 10.02.2014. 
riatic patients. We do believe that knowing patients' beliefs regarding their disease, we will be able to elaborate better management strategies not only regarding treatment efficacy but also helping them in coping with various aspects of psoriasis [6].

\section{Aim}

The aim of the study was to evaluate apprehension of the disease by psoriatic patients.

Table 1. Demographic and clinical characteristics of enrolled patients

\begin{tabular}{|c|c|}
\hline Parameter & Result \\
\hline \multicolumn{2}{|l|}{ Gender, $n$ : } \\
\hline Women & 36 \\
\hline Men & 64 \\
\hline \multicolumn{2}{|l|}{ Age [years]: } \\
\hline Mean \pm standard deviation & $47.3 \pm 15.8$ \\
\hline Range & $16-82$ \\
\hline \multicolumn{2}{|l|}{ Place of living, $n$ : } \\
\hline Village & 47 \\
\hline Town/city & 53 \\
\hline \multicolumn{2}{|l|}{ Educational status, $n$ : } \\
\hline Primary school & 10 \\
\hline Secondary school & 24 \\
\hline High school & 45 \\
\hline University & 21 \\
\hline \multicolumn{2}{|l|}{ Employment status, $n$ : } \\
\hline Employed & 48 \\
\hline Jobless/retired & 52 \\
\hline \multicolumn{2}{|l|}{ Smoking status, $n$ : } \\
\hline Non-smoker & 45 \\
\hline Smoker & 43 \\
\hline Ex-smoker & 12 \\
\hline \multicolumn{2}{|l|}{ Family history of psoriasis, $n$ : } \\
\hline Negative & 63 \\
\hline Positive & 37 \\
\hline \multicolumn{2}{|l|}{ Disease duration [years]: } \\
\hline Mean \pm standard deviation & $17.3 \pm 14.8$ \\
\hline Range & $0.1-59$ \\
\hline \multicolumn{2}{|l|}{ Age at disease onset [years]: } \\
\hline Mean \pm standard deviation & $29.6 \pm 17.6$ \\
\hline Range & $2-76$ \\
\hline \multicolumn{2}{|c|}{ Duration of the current exacerbation [months]: } \\
\hline Mean \pm standard deviation & $4.6 \pm 3.5$ \\
\hline Range & $0.5-12$ \\
\hline
\end{tabular}

\section{Material and methods}

\section{Patients' characteristics}

Patients were recruited consecutively from individuals admitted to the Department of Dermatology, Venereology and Allergology for the treatment of psoriasis exacerbation. All patients agreed voluntarily to participate in the study. A total of 100 subjects ( 36 women and 64 men) aged from 16 to 82 years (mean: $47.3 \pm 15.8$ years) with plaque type psoriasis were enrolled. Twenty-four (24\%) patients also suffered from psoriatic arthritis. Detailed characteristics of recruited subjects are demonstrated in Table 1.

\section{Study design}

Each participant underwent a careful physical examination and subsequently completed a questionnaire containing questions about demographic data, subjective psoriasis severity (mild, moderate, severe, very severe), factors inducing or aggravating psoriasis, influence of psoriasis on patients, patients' hopes and beliefs regarding the antipsoriatic treatment and the most bothersome symptoms of psoriasis. Assessment of objective disease severity was performed according to PASI [4].

\section{Statistical analysis}

All data were analyzed statistically using Statistica 9.0 (Statsoft, Krakow, Poland). The $\chi^{2}$ test with Yates correction, Student $T$ test, and Spearman rank correlation test were used where appropriate. Values of $p$ less than 0.05 were considered statistically significant.

\section{Results}

\section{Disease severity}

The disease severity according to PASI ranged between 1.5 and 48.0 points (mean: $17.1 \pm 10.0$ points). None of the analyzed demographic and clinical parameters (gender, age, living place, education, employment, smoking habit, family history of psoriasis, age at disease onset, psoriasis duration and duration of the current exacerbation) significantly influenced the objective severity of psoriasis (data not shown). Among the studied individuals, 4 patients assessed their psoriasis as mild, 41 as moderate, 47 as severe, and 8 as very severe. No significant differences were observed between the analyzed subgroups and self-assessed psoriasis severity. The correlation between the PASI scoring and subjective assessment of psoriasis severity was of the modest degree, albeit significant $(\rho=0.37, p<0.001$; Figure 1$)$.

\section{Influence of psoriasis on patients' life}

Patients who were interrogated about the influence of psoriasis on their life indicated that psoriasis had an enormous negative impact on their life and disturbed 
them very much (49\% of patients), decreased their self-esteem ( $40 \%$ of patients), had a huge negative impact on their economic condition (33\% of patients), caused problems in establishing relationships with other people (29\% of patients), and determined how other people perceived them (28\% of patients). In addition, $7 \%$ of subjects underlined that they felt stigmatized by psoriasis. On the other hand, $16 \%$ of subjects claimed that after so many years of being ill, psoriasis is unimportant for them and further $13 \%$ declared that the skin problem never influenced their life.

Patients saying that psoriasis decreased their self-esteem, were significantly younger $(43.5 \pm 15.0$ years vs. $49.8 \pm 15.8$ years, $p<0.05)$ and more frequently employed $(62.5 \%$ vs. $38.3 \%, p=0.03)$. Patient underlining that psoriasis had a huge impact on their economic condition more often had joint disease (39.4\% vs. $16.4 \%, p=0.02$ ), suffered from psoriasis significantly longer $(22.3 \pm 15.2$ years vs. $14.8 \pm 14.1$ years, $p=0.02)$, and had more severe disease (mean PASI: $20.4 \pm 10.8$ points vs. $15.5 \pm 9.2$ points, $p=0.02$ ). Moreover, patients assessing their psoriasis as severe or very severe, more often indicated the great influence of psoriasis on their life (0\% vs. $26.8 \%$ vs. $68.1 \%$ vs. $75 \%$ for mild, moderate, severe and very severe psoriasis, respectively, $p<0.001)$, underlined the problems of high costs of antipsoriatic treatment (0\% vs. $17.1 \%$ vs. $42.5 \%$ vs. $75 \%, p=0.001)$, and less often declared that psoriasis is unimportant for them (75\% vs. $17.1 \%$ vs. $12.8 \%$ vs. $0 \%, p<0.01)$. No other significant relationships were found between analyzed parameters and claims about the influence of psoriasis on patients' life.

\section{Causative and exacerbating factors}

Table 2 presents the data on the patients' opinion about factors inducing and exacerbating psoriasis. Emotional stress has been indicated as the most common factor both inducing as well as exacerbating psoriasis. Patients with positive family history of psoriasis more often indicated genetic factors as both inducing and exacerbating psoriasis (70.3\% vs. $22.2 \%, p<0.001$ and $43.2 \%$ vs. $9.7 \%, p<0.001)$. They also more commonly identified emotional stress as an aggravating parameter of psoriasis $(75.7 \%$ vs. $52.4 \%, p<0.05)$. Patients indicating that psoriasis is caused by genetic factors, were significantly younger ( $42.6 \pm 15.7$ years vs. $50.4 \pm 15.1$ years, $p=0.01$ ). In addition, subjects indicating stress as an important pathogenic parameter had significantly less severe disease (mean PASI: $15.0 \pm 9.6$ points vs. $19.2 \pm 10.0$ points, $p=0.03$ ). No other significant relationships were found in respect of factors inducing or exacerbating psoriasis.

\section{Opinions about disease prognosis and treatment}

Regarding the course and prognosis of psoriasis, $41 \%$ of patients indicated that their skin problem may disappear, but, for sure, it will come back, and 37\% said that

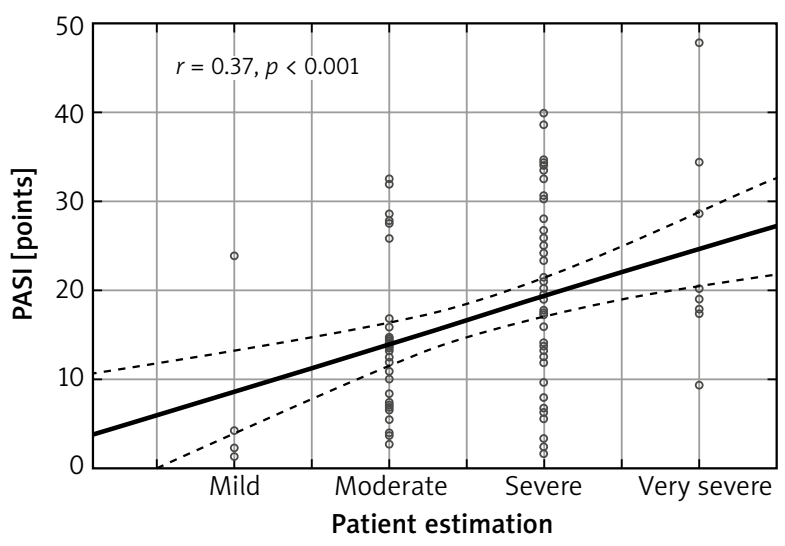

Figure 1. Comparison of the patients' self-assessment of psoriasis severity and PASI value

Table 2. Patients' opinions about factors inducing and exacerbating psoriasis

\begin{tabular}{lcc}
\hline Variable & $\begin{array}{c}\text { Factors inducing } \\
\text { psoriasis }\end{array}$ & $\begin{array}{c}\text { Factors } \\
\text { exacerbating } \\
\text { psoriasis }\end{array}$ \\
\hline Emotional stress & $49(49 \%)$ & $61(61 \%)$ \\
\hline Genetic factors & $40(40 \%)$ & $22(22 \%)$ \\
\hline Bacterial or viral infections & $21(21 \%)$ & $26(26 \%)$ \\
\hline Chance & $21(21 \%)$ & $7(7 \%)$ \\
\hline Environmental pollution & $15(15 \%)$ & $17(17 \%)$ \\
\hline Diet & $11(11 \%)$ & $21(21 \%)$ \\
\hline Poor healthcare & $5(5 \%)$ & $12(12 \%)$ \\
\hline Other & $14(14 \%)$ & $13(13 \%)$ \\
\hline Don't know & $18(18 \%)$ & $13(13 \%)$ \\
\hline
\end{tabular}

they will live with psoriasis for the whole life. However, a marked portion of patients believed that their disease will be cured in the future: $20 \%$ of participants expected a rapid complete cure of psoriasis, $6 \%$ hoped that the disease will last for a couple of months or years, but then disappear, and further $7 \%$ believed that they will suffer from psoriasis for many years, but eventually the disease will vanish. Fifteen subjects had no idea about the course of psoriasis. On the other hand, the majority of patients (56\%) knew that no curative therapy is currently available, but they had strong beliefs that such therapy will be developed in the future. Twenty-eight percent stated that their daily habits significantly influenced the disease course, and 22\% claimed that there are a number of things they can do to improve their psoriasis. On the other hand, $25 \%$ of patients were very pessimistic saying that nothing can be done as the psoriasis is an incurable disease. In addition, $17 \%$ believed that the treatment, 
which was used by them at the time of evaluation, would cure their psoriasis; $16 \%$ said that currently available options made the complete cure of psoriasis very probable, and $10 \%$ stated that the cure of psoriasis depends on a chance.

Among the analyzed variables, only positive family history of psoriasis and education level had some influence on the beliefs about psoriasis course. Patients with familiar background of psoriasis more frequently knew that psoriasis will have a relapsing course $(56.8 \%$ vs. $31.7 \%, p=0.02$ ) and less often expected a complete cure of the disease ( $8.1 \%$ vs. $27.0 \%, p<0.05)$. In addition, patients having only primary school education compared to the rest of patients more often expected that the treatment they used would cure the disease (50.0\% vs. $13.3 \%, p=0.01)$ and that their disease will last for several months or years, but then it will disappear (30.0\% vs. $3.3 \%, p<0.01)$. Patients claiming that psoriasis will last for their entire life were older $(52.2 \pm 16.5$ years vs. 44.3 \pm 14.7 years, $p=0.02$ ) and suffered longer from psoriasis ( $23.7 \pm 16.6$ years vs. $13.5 \pm 12.3$ years, $p<0.001)$. On the other hand, patients expecting a rapid cure of disease had experienced a later disease onset (at a mean age of $37.6 \pm 17.1$ years vs. $27.6 \pm 17.2$ years, $p=0.02$ ) and suffered from psoriasis for a significantly shorter time (11.2 \pm 10.2 years vs. $18.8 \pm 15.4, p<0.05$ ).

\section{Symptoms of psoriasis}

According to patients' opinions, the most burdensome symptoms of psoriasis were as follows: intense epidermal scaling (66\% of responders), itching (65\%), skin redness (51\%), burning (44\%), dandruff (38\%), nail abnormalities (37\%), pain (28\%), joint stiffness (24\%), sleeping difficulties (21\%), joint deformation (15\%), debility (11\%), fatigue (11\%), eye irritation (10\%), hair loss (9\%), oozing (7\%), and gastric problems related to antipsoriatic therapy (5\%). Employed persons more often underlined the problem of skin redness ( $64.6 \%$ vs. $38.5 \%, p<0.01)$. Older people more likely considered oozing (mean age: 58.6 \pm 9.9 years vs. $46.4 \pm 15.8$ years, $p<0.05$ ), eye irritation (mean age: $57.3 \pm 6.0$ years vs. $46.1 \pm 16.1$ years, $p=0.03$ ), joint stiffness (mean age: $56.6 \pm 9.5$ years vs. $44.3 \pm 16.2$ years, $p<0.001$ ) and deformation (mean age: $56.2 \pm 9.0$ years vs. $45.7 \pm 16.2$ years, $p=0.02$ ) as the most burdensome psoriasis symptoms, while epidermal scaling was less important for them (mean age: $43.9 \pm 15.2$ years vs. $53.8 \pm 15.0$ years, $p<0.01$ ). Furthermore, patients having a diagnosis of psoriatic arthritis more frequently indicated pain $(62.5 \%$ vs. $17.1 \%, p<0.001)$, joint stiffness (66.7\% vs. 10.5\%, $p<0.001)$ and joint deformations (50\% vs. 3.9\%, $p<0.001$ ) as the most bothersome symptoms of psoriasis. Patients with more severe disease more often underlined the problem of burning (mean PASI: 19.4 \pm 10.5 points vs. $15.3 \pm 9.2$ points, $p<0.05$ ), nail abnormalities (mean PASI: $19.8 \pm 9.6$ points vs. $15.4 \pm 9.9$ points, $p=$ 0.03), debility (mean PASI: $24.8 \pm 13.9$ points vs. $16.2 \pm 9.0$ points, $p<0.01$ ), fatigue (mean PASI: $24.1 \pm 12.6$ points vs. $16.3 \pm 9.3$ points, $p=0.01$ ), and pain (mean PASI: 21.5 \pm 12.5 points vs. $15.4 \pm 8.3$ points, $p<0.01$ ). In addition, patients with a longer disease duration more frequently indicated pain (mean disease duration: $22.1 \pm 17.0$ years vs. $15.4 \pm 13.5$ years, $p=0.04$ ), joint stiffness (mean disease duration: $24.9 \pm 17.9$ years vs. $14.9 \pm 12.9$ years, $p<0.01$ ) and eye irritation (mean disease duration: 24.1 \pm 12.6 years vs. $16.3 \pm 9.3$ years, $p=0.01$ ), as well as significantly less often skin redness (mean disease duration: $14.3 \pm 12.7$ years vs. $20.3 \pm 16.3$ years, $p=0.04$ ) as the most bothersome psoriasis ailments.

\section{Discussion}

Psoriasis is a disfiguring and stigmatizing skin disease, the course of which is punctuated by exacerbations and remissions. It is associated with problems of body image, self-esteem, feelings of stigma and shame [7]. In order to respond to difficulties that this disease may cause, many patients create their own cognitive model of their condition. This system of beliefs can be, of course, inaccurate, however, there is growing evidence that it directs attempts to cope with a condition and influences compliance with treatment. Thus, patients' opinions have important implications for the clinical management of psoriasis [5].

We have shown that some patients with psoriasis are not aware of the course of their disease and demonstrated false beliefs that their condition will be cured now or in the near future. Although we cannot exclude for sure that a curative treatment modality will be developed for psoriasis sometime in the future, such possibility seems to be rather unlikely taking into consideration our current understanding of psoriasis pathogenesis. For now a complete cure of psoriasis is an unmet and unrealistic expectation that can result, in such unaware patients, in a frustration from currently available therapies, which in most cases are only able to provide temporary relief from skin problems. Unrealistic expectations may thus cause dissatisfaction with antipsoriatic treatments leading to a poor adherence to prescribed therapies and resulting in even worse psoriasis improvement than could be achieved [8]. Therefore, it should be postulated that any newly diagnosed patient with psoriasis should receive detailed and proper information about the disease course, treatment options, exacerbating factors, etc. [9]. Such educational program might not only increase the awareness about psoriasis, but could also help to elaborate with patients the best treatment strategies they will comply with.

A number of studies analyzed and confirmed the negative impact of psoriasis on patients' well-being. Even limited skin lesions may result in a significant social withdrawal. Many psoriatic patients avoid going to the beach, public baths, swimming pools, hairdressers or 
even wearing dark clothes $[4,10]$. Because of the fear of being discriminated psoriasis causes a feeling of decreased self-esteem and problems in interpersonal relationships [11]. As mentioned by our patients, psoriasis is also linked with a substantial economic burden. For patients, costs of psoriasis therapy were estimated as high as $9-14 \%$ of their salary [12]. It was also shown that the more severe the psoriasis, the higher the costs of care are, and disease severity is correlated with the cost of treatment, time required for treatment, and time lost from work. Remarkably, about $20 \%$ of subjects declared that they had not enough money to pay for all prescribed antipsoriatic drugs, further contributing to the poor response to the treatment $[13,14]$.

While treating patients with psoriasis it should not be forgotten that not always disease aspects relevant to physicians are of the same importance for patients. Many patients underlined that subjective symptoms connected with psoriatic lesions, like itching, burning or even pain, are considered by them as the most bothersome aspects of their disease. Remarkably, these symptoms are frequently not well controlled with current antipsoriatic treatment [15] and they greatly contribute to quality of life impairment in this group of patients. Importantly, the relevance of certain psoriasis aspects are changing over time and older people handle their disease in a different way than young patients do $[16,17]$. Antipsoriatic therapy should be adjusted appropriately to better meet patients' needs and to improve their psychosocial well-being.

\section{Conclusions}

In the era of biological treatments, psoriasis still constitutes an important and difficult clinical problem. The disease causes significant psycho-social and economic burden for patients and their relatives. Any attempts should be made to widen understanding of the disease among patients and their families in order to make the patients' expectations realistic and to improve adherence to antipsoriatic treatments. Furthermore, current treatment strategies should better defeat not only psoriatic lesions, but also control subjective symptoms greatly affecting patients' well-being.

\section{References}

1. Poulin Y, Papp KA, Wasel NR, et al. Canadian online survey to evaluate awareness and treatment satisfaction in individuals with moderate to severe plaque psoriasis. Int I Dermatol 2010; 49: 1368-75.

2. Stern RS, Nijsten T, Feldman SR, et al. Psoriasis is common, carries a substantial burden even when not extensive, and is associated with widespread treatment dissatisfaction. J Investig Dermatol Symp Proc 2004; 9: 136-9.

3. Nijsten J, Margolis DJ, Feldman SR, et al. Traditional systemic treatments have not fully met the needs of psoriasis patients: results from a national survey. J Am Acad Dermatol 2005; 52: 434-44.
4. Hrehorów E, Salomon J, Matusiak L, et al. Patients with psoriasis feel stigmatized. Acta Derm Venereol 2012; 92: 67-72.

5. Fortune D, Richards H, Main C, Griffiths C. What patients with psoriasis believe about their condition. J Am Acad Dermatol 1998; 39: 196-201.

6. Scharloo M, Kaptein AA, Weinman J, et al. Patients' illness perceptions and coping as predictors of functional status in psoriasis: a 1-year follow-up. Br J Dermatol 2000; 142: 899907.

7. Poot F, Sampogna F, Onnis L. Basic knowledge in psychodermatology. J Eur Acad Dermatol Venereol 2007; 21: 227-34.

8. Carroll CL, Feldman SR, Camacho FT, Balkrishnan R. Better medication adherence results in greater improvement in severity of psoriasis. Br J Dermatol 2004; 151: 895-7.

9. Larsen MH, Hagen KB, Krogstad AL, et al. Limited evidence of the effects of patient education and self-management interventions in psoriasis patients: a systematic review. Patient Educ Couns 2014; 94: 158-69.

10. Hrehorów E, Reich A, Szepietowski J. Quality of life in patients with psoriasis: relationship with pruritus, stress and symptoms of depression. Dermatol Klin 2007; 9: 19-23.

11. Sampogna F, Gisondi P, Tabolli S, et al. Impairment of sexual life in patients with psoriasis. Dermatology 2007; 214: 144-50.

12. Lundberg L, Johannesson M, Silverdahl M, et al. Quality of life, health-state utilities and willingness to pay in patients with psoriasis and atopic eczema. Br J Dermatol 1999; 141: 1067-75.

13. Gupta M, Gupta A. Age and gender differences in the impact of psoriasis on quality of life. Int I Dermatol 1995; 34: 700-3.

14. Bewley A, Page B. Maximizing patient adherence for optimal outcomes in psoriasis. J Eur Acad Dermatol Venereol 2011; 25 Suppl. 4: 9-14.

15. Dawn A, Yosipovitch G. Treating itch in psoriasis. Dermatol Nurs 2006; 18: 227-33.

16. Reich A, Hrehorów E, Szepietowski JC. Pruritus is an important factor negatively influencing the well-being of psoriatic patients. Acta Derm Venereol 2010; 90: 257-63.

17. Ljosaa TM, Rustoen T, Mörk C, et al. Skin pain and discomfort in psoriasis: an exploratory study of symptom prevalence and characteristics. Acta Derm Venereol 2010; 90: 39-45. 